\title{
Hierarchical electrospun tendon-ligament bioinspired scaffolds induce changes in fibroblasts morphology under static and dynamic conditions
}

Alberto Sensini ${ }^{1}$, Luca Cristofolini ${ }^{1,2}$, Andrea Zucchelli ${ }^{1}$, Maria Letizia Focarete ${ }^{2,3}$, Chiara Gualandi ${ }^{3,4}$, Arianna De Mori ${ }^{5}$, Alexander Kao ${ }^{6}$, Marta Roldo $^{5}$, Gordon Blunn ${ }^{5}$, Gianluca Tozzi ${ }^{6}$

1 Department of Industrial Engineering, Alma Mater Studiorum-University of Bologna, I-40131 Bologna, Italy

2 Health Sciences and Technologies - Interdepartmental Center for Industrial Research (CIRI-HST), Alma Mater Studiorum-University of Bologna, I-40064 Ozzano dell'Emilia, Bologna, Italy

3 Department of Chemistry 'G. Ciamician' and National Consortium of Materials Science and Technology (INSTM, Bologna RU), Alma Mater StudiorumUniversity of Bologna, I-40126 Bologna, Italy

4 Advanced Mechanics and Materials - Interdepartmental Center for Industrial Research (CIRI-MAM), Alma Mater Studiorum-University of Bologna, I-40123 Bologna, Italy

5 School of Pharmacy and Biomedical Science, University of Portsmouth - St Michael's Building, White Swan Road, Portsmouth PO1 2DT, United Kingdom

6 Zeiss Global Centre, School of Mechanical and Design Engineering, University of Portsmouth, Portsmouth PO1 3DJ, United Kingdom

Corresponding author:

Gianluca Tozzi

Zeiss Global Centre

School of Mechanical and Design Engineering

University of Portsmouth

PO1 3DJ Portsmouth, United Kingdom

e-mail: gianluca.tozzi@port.ac.uk 


\section{Second abstract}

2 To enhance the regeneration of injured tendons and ligaments cells need to growth on

3 dedicated structures (scaffolds) with mechanical properties and a fibrous morphology

4 similar to the natural tissue. In particular, the morphological organization of scaffolds is

5 fundamental in leading cells to colonize them, regenerating the collagen extracellular

6 matrix. Electrospinning is a promising technique to produce fibers with a similar to the

7 human collagen fibers, suitable to design complex scaffolds able to guide cells in the

8 reconstruction of the natural tissue. Moreover, it is well established that the cyclic

9 stretching of these scaffolds inside dedicated systems called bioreactors, can speed up

10 cells growth and their shape modification. The aim of the present study was to

11 investigate how hierarchically structured electrospun scaffolds, made of resorbable

12 material such as poly(L-lactic acid) and collagen, could induce morphological changes

13 in human fibroblasts, while cultured during static and dynamic conditions. These

14 scaffolds were composed by an external electrospun membrane that grouped inside it a

15 ring-shaped bundle, made of axially aligned nanofibers, resembling the morphological

16 arrangement of tendon and ligament tissue. After 7 days of parallel cultures, the

17 scaffolds were investigated using the following imaging techniques: (i) high-resolution

18 X-ray tomography (XCT); (ii) scanning electron microscopy (SEM); (iii) fluorescence

19 microscopy; (iv) histology. The results showed that fibroblasts were able to grow on the

20 external nanofibrous sheath of the static scaffolds, by elongating themselves along their

21 circumference. The dynamic cultures revealed instead a preferential axial orientation of

22 fibroblasts grown on the external sheath. The aligned nanofiber bundles inside the

23 hierarchical scaffolds allowed an axial distribution of the fibroblasts along the

24 nanofibers direction. This study has demonstrated that the electrospun hierarchically 
25 structured scaffolds investigated can modify the fibroblasts morphology both in static

26 and dynamic conditions, in relation with the direction of the applied loads.

\section{Summary}

28 The regeneration of injured tendons and ligaments is challenging since the scaffolds

29 needs proper mechanical properties and a biomimetic morphology. In particular, the

30 morphological arrangement of scaffolds is a key point to drive the cells growth to

31 properly regenerate the collagen extracellular matrix. Electrospinning is a promising

32 technique to produce hierarchically structured nanofibrous scaffolds able to guide cells

33 in the regeneration of the injured tissue. Moreover, the dynamic stretching in bioreactors

34 of electrospun scaffolds had demonstrated to speed up cell shape modifications in vitro.

35 The aim of the present study was to combine different imaging techniques such as high-

36 resolution X-ray tomography (XCT), scanning electron microscopy (SEM),

37 fluorescence microscopy and histology to investigate if hierarchically structured poly(L-

38 lactic acid) and collagen electrospun scaffolds can induce morphological modifications

39 in human fibroblasts, while cultured in static and dynamic conditions. After 7 days of

40 parallel cultures, the results assessed that fibroblasts had proliferated on the external

41 nanofibrous sheath of the static scaffolds, elongating themselves circumferentially. The

42 dynamic cultures revealed a preferential axial orientation of fibroblasts growth on the

43 external sheath. The aligned nanofiber bundles inside the hierarchical scaffolds instead,

44 allowed a physiological distribution of the fibroblasts along the nanofiber direction.

45 Inside the dynamic scaffolds, cells appeared thinner compared with the static

46 counterpart. This study had demonstrated that hierarchically structured electrospun

47 scaffolds can induce different fibroblasts morphological modifications during static and 48 dynamic conditions, modifying their shape in the direction of the applied loads. 
50 Keywords:

51 Electrospinning, Hierarchical Scaffolds, High-Resolution X-Ray Tomography, Cell

52 Culture, Dynamic Cell Culture, Cell Morphology, Tissue Engineering, Tendons and 53 Ligaments.

54 


\section{Introduction}

56 The challenge of the innovative three-dimensional scaffolds, suitable for tendon and

57 ligament regeneration, is to strictly reproduce the native tissue mechanical properties 58 and hierarchical morphology (Alshomer et al., 2018; Cheng et al., 2015; Goulet et al., 59 2014; Kuo et al., 2010). The morphological arrangement of the scaffold is fundamental 60 to correctly drive cell proliferation and growth, during collagen extracellular matrix 61 regeneration. It has been shown that fibroblasts and tenocytes shape is strictly dependent 62 on the specific site of growth in vivo: cells that colonize tendon and ligament membranes

63 (made of randomly arranged collagen fibrils), tend to spread their bodies; conversely, 64 cells in the internal volume of these tissues appear elongated in the direction of the 65 axially oriented fibrils (Kannus, 2000; Kastelic et al., 1978; Murphy et al., 2016). 66 Several manufacturing approaches to produce fibrous scaffolds inspired to tendons or

67 ligaments have been investigated in literature, among these electrospinning technology 68 is the most promising (Sensini \& Cristofolini, 2018). Thanks to the possibility to obtain 69 nanoscale fibers with different spatial arrangements, electrospun scaffolds have 70 demonstrated enhancement of cellular orientation in the fibers direction (Bosworth \& 71 Downes, 2011; Denchai et al., 2018). Furthermore, several studies have confirmed the 72 possibility to speed up cell proliferation and elongation on the electrospun scaffolds with 73 a simplified shape, such as flat mats, bundles or yarns, by uniaxially stretching the 74 constructs in a bioreactor (Bosworth et al., 2014; Wu et al., 2017; Xu et al., 2014; 75 Youngstrom \& Barrett, 2016). These simple designs allow for convenient 76 documentation of changes in cellular shape using standard techniques, such as scanning

77 electron microscopy (SEM), fluorescent microscopy or histology. Despite the high78 quality of images and the cellular information obtainable, these gold-standard methods 79 have shown some limitation when applied to the study of the cell morphology on 
80 complex three-dimensional scaffolds (Leferink et al., 2016). SEM images can achieve a

81 high-resolution, but are limited to the surface of the structures. Fluorescent techniques,

82 such as fluorescent or confocal microscopy, allow an accurate identification of the shape

83 of cells, but are strongly limited by possible autofluorescent effects of the nanofibers,

84 especially if they are composed by natural polymers such as collagen (Sensini et al.,

85 2018). Moreover, these techniques do not allow easy visualization if the structure

86 investigated is not planar, making the investigation of three-dimensional scaffolds

87 challenging. On the other hand, histology allows a clear identification of the cellular

88 components, even in case of three-dimensional shapes, but it typically produces a bi-

89 dimensional view of the specimens and of the cells inside. This limits a correct definition

90 of the cellular shape. Moreover, during the slicing and washing procedure, in particular

91 for the electrospun materials, it is easy to damage parts of the scaffold losing the related

92 information. A possible solution to overcome these limitations is offered by high-

93 resolution X-ray computed tomography (XCT). However, due to the low X-ray

94 attenuation of the polymeric nanofibers, XCT investigation of electrospun materials is

95 particularly challenging. This problem is especially true in the case of collagenous

96 materials (Balint et al., 2016; Zidek et al., 2016). Recent studies have defined dedicated

97 protocols to overcome such limitations even in case of submicron voxel sizes (Bosworth

98 et al., 2014; Sensini et al., 2018). Furthermore, Bradley et al. have defined a procedure

99 to document, by using a laboratory XCT, cell infiltration inside electrospun mats of

100 random microfibers (Bradley et al., 2017). However, to the best of our knowledge, no

101 work has ever tried to investigate the cell growth and infiltration in complex three-

102 dimensional electrospun nanofibrous scaffolds by combining XCT and other different

103 imaging techniques. This approach could be fundamental to analyze how the different

104 elements of the scaffolds can induce cellular morphological modifications. 
105 The aim of the present study was to compare the fibroblast morphological modifications 106 during static and dynamic culture protocols on complex electrospun scaffolds. Cells

107 were seeded on three-dimensional electrospun nanofibrous hierarchically structured 108 scaffolds made of a poly(L-lactic acid) (PLLA) and collagen (Coll) blend. Different 109 imaging techniques including high-resolution X-ray tomography (XCT), scanning 110 electron microscopy (SEM), fluorescent microscopy and histology were employed 111 confirming different cellular modifications in shape and orientation during static and 112 dynamic conditions of culture.

\section{Materials and methods}

114 In order to investigate the morphologically changes in the fibroblasts shape, electrospun

115 PLLA/Coll nanofibrous hierarchically structured scaffolds were produced (Figure 116 1(A)). The scaffolds were seeded with human fibroblasts and cultured in different 117 conditions for 7 days: two of each in static conditions, while the other two were stretched 118 two times in a bioreactor for 1 hour each (Figure 1(B)). At the end of the culture, the 119 specimens were cut in pieces and investigated with different imaging techniques (Figure $1201(\mathrm{C}))$.

\section{Hierarchical electrospun scaffolds production}

122 In order to reproduce the morphology of tendon and ligament fibrils and fascicles 123 (Kannus, 2000; Murphy et al., 2016), electrospun bundles (cross-sectional diameter = $124550-650 \mu \mathrm{m}$ ) of aligned nanofibers (cross-sectional diameter of the nanofibers $=$ $1250.36 \pm 0.06 \mu \mathrm{m})$ of a PLLA/Coll-75/25 (w/w) blend were produced as previously 126 described (Sensini et al., 2017, 2018). To obtain the bundles the following 127 electrospinning parameters were used: a rotating drum collector (peripheral speed $=22.8$ $128 \mathrm{~m} \mathrm{~s}^{-1}$ ); the polymer solution was delivered through two needles (internal diameter 0.51 
$129 \mathrm{~mm}$ ); room temperature (RT) and relative humidity $20-30 \%$; applied voltage $=22 \mathrm{kV}$;

130 feed rate $=0.5 \mathrm{~mL} \mathrm{~h}^{-1}$, electrospinning time $=2$ hours; needles-collector distance $=200$

$131 \mathrm{~mm}$; the sliding spinneret with the two needles had an excursion of $120 \mathrm{~mm}$, with a

132 sliding speed of $1200 \mathrm{~mm} \mathrm{~min}^{-1}$.

133 To reproduce the structure of a whole tendon or ligament (Kastelic et al., 1978; Murphy

134 et al., 2016), each bundle was pulled out from the drum, obtaining a ring-shaped

135 structure that was twisted in the middle and bent over itself. Then, each assembly was 136 covered with an electrospun epitenon/epiligament-like sheath, as previously described

137 (WO 2018/229615 A1, 2018; Sensini et al., 2019; Sensini et al., 2019). The scaffolds

138 were finally crosslinked with a mixture of $N$-(3-dimethylaminopropyl)- $N^{\prime}-$

139 ethylcarbodiimide hydrochloride (EDC) and $N$-hydroxysuccinimide (NHS) (Sigma-

140 Aldrich, USA) as previously described (Alberto Sensini et al., 2018) (cross-sectional

141 diameter $=1.46 \pm 0.08 \mathrm{~mm}$; length of the scaffolds $=89.4 \pm 2.1 \mathrm{~mm})$. Four hierarchical

142 scaffolds were produced (Figure 1).

143 Cell seeding

144 The four hierarchical scaffolds were sterilized by immersion in $70 \%(\mathrm{v} / \mathrm{v})$ ethanol (Acros

145 Organics, Thermo Fisher Scientific, BEL) for 1 hour, washed in sterile PBS (Thermo

146 Fisher Scientific, USA) three times to remove any remaining ethanol and equilibrated in

147 complete medium for 24 hours. The complete medium was obtained by mixing

148 Dulbecco Modified Eagle Medium (DMEM) (i.e. 4.5 g/L D-Glucose, with

149 GlutaMAX ${ }^{\mathrm{TM}}$ and Pyruvate) (Thermo Fisher Scientific, USA), 10\% foetal bovine serum

150 (Thermo Fisher Scientific, USA) and 1\% (v/v) penicillin/streptomycin solution (Thermo 151 Fisher Scientific, USA). 
152 Human foreskin fibroblasts (Hs27) were cultivated with complete medium at $37^{\circ} \mathrm{C}$ in a

153 humid atmosphere with $5 \% \mathrm{CO}_{2}$. Medium was refreshed three times a week and cells

154 were used between passage 4 and 6.

155 To perform the test, cells were seeded at $2.0 \times 10^{5}$ cells/scaffold. In particular, cells were

156 suspended in 350 microliters of complete medium and seeded, using a syringe with a

157 25G needle: half volume was seeded on one side of the scaffold, then the scaffold was

158 turned $180^{\circ}$ and the other half volume was seeded on the other side. The seeding was

159 carried out in a sterile petri dish. After 45 minutes in an incubator at $37^{\circ} \mathrm{C}$ and $5 \% \mathrm{CO}_{2}$,

160 each hierarchical scaffold was transferred into one low adherence T25 flask each and

161 covered with $5 \mathrm{ml}$ of complete medium to allow for cell proliferation.

162 In order to avoid potential artefacts caused by the relevant amount of medium during the

163 dynamic cultures (see below) and the total length of the specimens, quantitative data

164 regarding the cell viability were not reported.

165 After 7 days of culture, the hierarchical scaffolds were fixed for 48 hours in $4 \%$

166 paraformaldehyde (PFA, Sigma-Aldrich, Saint Louis, USA) in PBS (at $4^{\circ} \mathrm{C}$ ). Then, each

167 specimen was cut in the center and divided in two equal sections: one half for the $168 \mathrm{SEM} / \mathrm{XCT}$ imaging; the other was cut in two additional pieces for fluorescence 169 microscopy and histology (Figure 1(C)).

\section{Dynamic cultures in bioreactor}

171 The dynamic culture was carried out on two hierarchical scaffolds by using a 172 commercial bioreactor (MCB1, CellScale, CAN). Before each stretching session, the 173 bioreactor was sterilized by washing the test chamber in ethanol $70 \%(\mathrm{v} / \mathrm{v})$ and sterilized 174 by means UV radiations under a fume hood for an hour. To transmit a uniaxial 175 stretching, the hierarchical scaffolds were hooked between the stainless-steel actuator of 
176 the bioreactor and a custom-made 3D printed pin of acrylonitrile butadiene styrene 177 (ABS) (ABS-M30, Stratasys, USA). During each session, the specimens were covered 178 with $150 \mathrm{ml}$ of complete medium and stimulated for 1 hour with $4 \mathrm{~mm}$ of displacement 179 (corresponding at a strain of approximately 5\%) at a frequency of $1 \mathrm{~Hz}$ (3600 cycles).

180 These parameters were chosen in accordance with the literature (Bosworth et al., 2014).

181 Each of the two scaffolds was stretched two times during the 7 days of culture (i.e. at 182 day three and day six of culture). After each bioreactor session, the dynamic specimens 183 were put in T25 flasks with $5 \mathrm{ml}$ of medium and left in static conditions for two days.

\section{$184 \quad$ Static cultures}

185 Parallelly, as a control for the dynamic specimens, two hierarchical scaffolds were 186 cultured for 7 days in T25 flasks with $5 \mathrm{ml}$ of medium, changing the medium at day 3 187 and six of culture.

189 To evaluate the full-field fibroblast distribution, morphology and the hierarchical 190 arrangement in the scaffolds, an XCT investigation was performed.

191 Firstly, after fixing with PFA, the scaffolds specimens for XCT were washed three times

192 in PBS. Specimens were post-fixed with osmium tetroxide (Sigma-Aldrich, USA) for 1 193 hour and then dehydrated in ethanol (v/v) 30\%, 50\%, 70\%, 90\%, 95\% and $100 \%$ for 1 194 hour for each step (the 100\% step was repeated twice). Then the specimens were 195 dehydrated in acetone for 20 minutes. The specimens were chemically dried using a 196 mixture of hexamethyldisilazane (HMDS) (Sigma-Aldrich, USA) and ethanol in 197 different (v/v) ratios: (i) HMDS:ethanol = 1:2 (v/v) and (ii) HMDS: ethanol = 2:1 (v/v) 198 for 20 minutes each. An additional step was performed in HMDS 100\% until dry. 
199 To avoid imaging artifacts resulting from micromovements, the specimens were fixed

200 in custom-made plastic masks adapted from (Sensini et al., 2018).

201 The two dynamic and static specimens of the hierarchical scaffolds were scanned with

202 a laboratory XCT system (Xradia 520 Versa, Zeiss X-ray Microscopy, USA), with the

203 following parameters:

204 (i) Voxel size $=1.6$ micrometers (i.e. overview of the specimens): $40 \mathrm{kV}$ voltage, 2

205 W power, 49 microampere tube current, 10 sec. exposure time.

206 (ii) Voxel size $=0.5$ micrometers (i.e. $z o o m$-in on the fibroblasts): $40 \mathrm{kV}$ voltage, 2

207 W power, 50 microampere tube current, 30 sec. exposure time.

208 All the XCT images, were reconstructed using the Scout-and-Scan Reconstructor

209 software (Zeiss, USA), and were visualized using XM3DViewer1.2.8 software (Zeiss,

210 USA).

211 SEM imaging

212 After the XCT investigation, in order to confirm the fibroblasts presence, the XCT

213 specimens were removed from the masks and prepared for the SEM imaging. Each

214 specimen was cut in two pieces: one was longitudinally opened with a scalpel to

215 investigate the fibroblasts on the internal bundles, while the other was left intact to

216 investigate the fibroblasts on the electrospun sheath. Scanning Electron Microscopy

217 (SEM) (Philips 515 SEM, NL) observations were carried out using an accelerating

218 voltage of $15 \mathrm{kV}$ and specimens were gold sputtered. 
221 In order to quantify the orientation of the nanofibers of the scaffolds, the Directionality 222 plugin of ImageJ was used (Liu, 1991; Schindelin et al., 2012; Schneider et al., 2012).

223 This approach quantifies the distribution of nanofibers within a given angle from the 224 axis of the specimen. The analysis was performed using a Local Gradient Orientation 225 method following a procedure previously applied (Sensini et al., 2018).

226 To assess the orientation of the bundles inside the hierarchical assemblies, a full volume 227 investigation was performed applying the procedure to all the slices of the XCT stack 228 (voxel size $=1.6$ micrometers), after reslicing. In order to list also the orientation of the 229 nanofibers in a single crosslinked PLLA/Coll-75/25 bundle, the Directionality analysis 230 data on a XCT scan from a previous study were reported (Sensini et al., 2018).

231 To quantify the orientation of the nanofibers in the electrospun sheath the Directionality 232 analysis was performed on a stack of 5 SEM surface images (magnification $=8000 \mathrm{x}$ ) 233 derived from (Sensini et al., 2018).

234 In order to investigate the preferential orientation of the fibroblasts on the external 235 sheath, in static and dynamic conditions of culture, a Directionality investigation was 236 performed on a stack of 2 fluorescent images for each condition of culture (see below) 237 (magnification $=20 x$ ) adapted from (Sensini et al., 2018; Tseng et al., 2013). As the 238 nuclei are better visible and are stretched in the same direction as the cell itself, the 239 analysis was based on the alignment of the nuclei. Firstly, to enhance visibility of the 240 cells nuclei the fluorescent images were segmented, using ImageJ. Over each segmented 241 image, a mask was produced onto which lines were drawn of the same length and 242 orientation of the longest axis of each nucleus. Finally, the masks were analyzed with 243 Directionality as previously described. 
246 After PFA fixing, specimens were washed with PBS and put in $3 \mathrm{ml}$ of Triton-X (Sigma-

247 Aldrich, USA) $0.1 \%(\mathrm{v} / \mathrm{v})$ for $15 \mathrm{~min}$. Then, the scaffolds were washed 3 times with 248 PBS, before being treated with 1\% (v/v) bovine serum albumin (BSA) (Sigma-Aldrich, 249 USA) in PBS, for 1 hour. Then, the specimens were washed twice with sterile PBS. 250 Phalloidin Dylight 550 (Thermo Fisher Scientific, USA) (2 units/ml in PBS) was added 251 to each sample before incubation for $90 \mathrm{~min}$ at RT. Then the specimens were washed 252 two times with PBS and DAPI (Sigma Aldrich, USA) $(2 \mu \mathrm{g} / \mathrm{ml})$ was added and 253 incubated for $20 \mathrm{~min}$ in the dark, at RT. The specimens were stored at $4{ }^{\circ} \mathrm{C}$ in petri dishes 254 containing sterile PBS to prevent specimen dehydration. Finally, the external surfaces 255 of the were imaged using a fluorescent microscope (Axio Imager Z1, Zeiss, USA) 256 equipped with a camera (Hamamatsu HR, Hamamatsu, JAP) and a color camera 257 (AxioCam MRc, Zeiss, USA) too. Images were processed by Volocity 6.3 software 258 (Quorum Technologies Inc, UK).

\section{Histology}

260 The specimens for the haematoxylin and eosin staining were fixed in 4\% (v/v) PFA/PBS 261 overnight. PFA fixed specimens were processed into paraffin (Histosec $₫$, Merck, 262 Darmstadt, GER), using a dedicated embedder (EG1150 H, Leica, Wetzlar, GER) and 263 sectioned (slices thickness $=5 \mu \mathrm{m}$ ) using a microtome (RM2235, Leica, GER). The 264 specimens were sectioned parallelly to their longitudinal axis. Sections were 265 deparaffinized and incubated in hematoxylin (Sigma-Aldrich, USA) and then in 266 alcoholic eosin (Sigma-Aldrich, USA) for 5 min respectively. Finally, sections were 267 differentiated, dehydrated in graded series of ethanol, and mounted in dibutyl phthalate 268 xylene (DPX) (Sigma-Aldrich, USA) using glass coverslips. The histological slices of 
269 the scaffolds were imaged using a microscope (Diaplan, Leitz, GER) and processed with

270 the Image-Pro Plus 6 software (Media Cybernetics, UK).

271 Cell morphology

272 In order to quantify the dimensions of cells (the length, i.e. the preferential direction of

273 elongation of the cellular body; width and thickness), in the different conditions of

274 culture, measurements of cells bodies were performed using ImageJ on the different

275 images acquired. The cell length and width were estimated from XCT, fluorescence,

276 SEM and histological images (static cultures: $\mathrm{n}=20$ cells for the length; $\mathrm{n}=27$ cells for

277 the width; dynamic cultures: $n=8$ cells for the length; $n=4$ cells for the width), while

278 the cell thickness was measured using SEM and histological images (static cultures: $\mathrm{n}=$

2797 cells; dynamic cultures: $\mathrm{n}=5$ cells). The mean (three measurements for each cell) of

280 each parameter was used to produce the final mean and standard deviation of each

281 dimension.

\section{Results}

283 Morphological investigation of the hierarchical scaffolds

284 To investigate the orientation of the nanofibers and bundles in the different levels of the

285 hierarchical scaffolds, a Directionality analysis was performed (Figure 2). The

286 Directionality analysis confirmed the preferential axial orientation of the nanofibers in

287 the bundles, with a predominant peak of $31.4 \pm 2.82 \%$ in the range of $0^{\circ}-3^{\circ}$ from the

288 bundle axis, and a decrescent distribution (Sensini et al., 2018). A small amount of

289 nanofibers $(0.55 \pm 0.08 \%)$ was perpendicular to the bundle $\left(87^{\circ}-90^{\circ}\right)$. The Directionality

290 investigation showed that the nanofibers of the sheaths for the hierarchical assemblies

291 had a slight preferential circumferential orientation: more than $31 \%$ of the nanofibers 
292 fell in the range of $66^{\circ}-90^{\circ}$. The preferential axial of alignment of the bundles inside the

293 hierarchical scaffolds was confirmed by a predominant peak of $61.6 \% \pm 9.43 \%$ in the

294 range of $0^{\circ}-3^{\circ}$, and a decrescent distribution.

\section{Fibroblasts morphology from XCT investigation}

296 The specimens mounting setup for the XCT scans successfully prevented the artefacts

297 of micromovements, permitting to obtain high-resolution images after the three-

298 dimensional reconstruction (Figure 3). The hierarchical scaffolds were homogeneous,

299 and the internal bundles strongly grouped by the electrospun sheath. The cells fixation

300 and dehydration procedure enabled visualization of the fibroblasts growth on the

301 hierarchical scaffolds (Figure 3). The reconstructions with a $1.6 \mu \mathrm{m}$ voxel size provided

302 an overview of the specimens (Figure 3(A)). Zooming on the sheath at $0.5 \mu \mathrm{m}$ voxel

303 size, fibroblasts were clearly distinguishable (Figure 3(B)). On the sheath of static

304 specimens, fibroblasts were circumferentially oriented along the axis of the scaffolds,

305 spreading their bodies also along the scaffold longitudinal axis (Figures 3(AI, AII) and

$3063(\mathrm{BI}, \mathrm{BII}))$. On the sheath of dynamic specimens, the fibroblasts were thinner and less

307 wide (see below), with increasing axial orientation compared to the static ones (Figure

308 3(AIII, AIV) and 3(BIII, BIV)). In the internal bundles, due to the high-alignment of the

309 nanofibers and the elongated shape of the fibroblasts, cell detection was not possible.

310 Fibroblasts morphology from SEM investigation

311 The SEM images obtained for the same specimens used for the XCT scans and are

312 shown in Figure 4. Despite the preferential random arrangement of the sheath

313 nanofibers, the static fibroblasts showed a circumferential orientation with spread bodies

314 (Figure 4(AI, II)), while cells on the dynamic specimens were thinner and preferentially

315 elongated axially to the hierarchical scaffolds (Figure 4(BI, II)). The SEM investigation 
316 also assessed the fibroblasts infiltration inside the hierarchical scaffolds, both in static

317 and dynamic conditions. In both test conditions the internal fibroblasts appeared

318 elongated and distributed axially aligned with the bundles nanofibers (Figure 4(AIII,

319 AIV) and (BIII, BIV)). In the dynamic specimens the fibroblast appeared thinner

320 compared to the static counterpart (Figure 4(BIII, IV)).

321 Fibroblasts morphology from fluorescence microscopy investigation

322 The fluorescence microscopy results are reported in Figure 5. On the static specimens,

323 fibroblasts were again circumferentially oriented on the nanofibrous sheath (the nuclei

324 were also ovalized in the transversal direction of the scaffolds), with spread bodies

325 (Figure 5(A)). In the sheath of dynamic specimens, the fibroblasts appeared more axially

326 aligned compared with the static ones (Figure 5(B)).

327 The Directionality analysis of the cells nuclei performed on the electrospun sheaths

328 revealed that, in static specimens, the $71.1 \%$ of cells were oriented in a range of $72^{\circ}-90^{\circ}$

329 (Figure 6). In the dynamic specimens instead, the $53.4 \%$ of cells were oriented in the 330 range of $0^{\circ}-18^{\circ}$ (Figure 6 ).

331 The fluorescence investigation in the internal bundles was not possible due to a low

332 infiltration of the fluorescent reagents.

\section{Fibroblasts morphology from histological investigation}

334 The histological investigation outcomes are showed in Figure 6. The axial slices of the

335 hierarchical scaffolds obtained, had cut transversally the fibroblasts grown on the 336 electrospun sheaths (Figure 7(AI, II) and 7(BI, II)). In both the static and dynamic 337 specimens, the fibroblasts appeared preferentially circumferentially arranged, due to the 338 reduced axial elongation of their bodies. Moreover, on the dynamic specimens, the 
339 fibroblasts were thinner than on the static ones (according to their progressive extension

340 in the axial direction). Cells also infiltrated inside the hierarchical scaffolds aligning

341 themselves in the nanofibers direction (Figure 7(AIII, IV) and 7(BIII, IV)). The

342 fibroblasts in the dynamic specimens appeared thinner compared to the static

343 counterpart.

\section{Quantification of cells morphology}

345 The quantification of cells morphology revealed that in the static specimens, the cells 346 on the electrospun sheaths had a length of $72.1 \pm 27.9$ micrometers, a width of $18.8 \pm 15.3$

347 micrometers and a thickness of $3.2 \pm 0.8$ micrometers; in the internal bundles, the cells 348 showed a length of $26.8 \pm 9.9$ micrometers, a width of $2.5 \pm 1.6$ micrometers and a 349 thickness of $1.5 \pm 0.2$ micrometers. In the dynamic specimens, the cells on the sheath had 350 a length of $77 \pm 52.4$ micrometers, a width of $8.9 \pm 6.9$ micrometers and a thickness of 351 1.9 \pm 0.6 micrometers; on the internal bundles the cells had a length of $21.4 \pm 8.5$ 352 micrometers, a width of $2.6 \pm 1.3$ micrometers and a thickness of $1.2 \pm 0.8$ micrometers.

\section{Discussion}

354 To produce an electrospun scaffold suitable for tendon and ligament tissue engineering, 355 proper mechanical properties need to be combined to a biomimetic hierarchical 356 structure. These properties are mandatory to transmit physiological loads to the cells, 357 enabling their proper infiltration and growth inside the scaffolds. The aim of this study 358 was to investigate an innovative electrospun PLLA/Coll-75/25 hierarchically structured 359 scaffold, using different imaging techniques, in order to evaluate its ability to guide the 360 fibroblasts growth in static and dynamic conditions. The hierarchical scaffolds were 361 assembled by wrapping a PLLA/Coll-75/25 ring-shaped bundles of axially aligned 
362 nanofibers, with an electrospun PLLA/Coll-75/25 sheath of randomly oriented

363 nanofibers. The scaffolds nanofibers and bundles were in the same size range of collagen

364 fibrils and fascicles reported in literature (Kastelic et al., 1978). The Directionality

365 analysis confirmed that bundles nanofibers, as well as bundles themselves, were axially

366 aligned with the hierarchical scaffolds, while the nanofibers of the sheath showed a

367 slightly circumferential orientation (Figure 2). In this way the hierarchical structure of a

368 whole tendon or ligament was reproduced (Kastelic et al., 1978; Murphy et al., 2016).

369 In order to evaluate the morphological changes in the cell shape induced by the

370 hierarchical scaffolds, Hs27 fibroblasts were seeded on them for 7 days, comparing a

371 static culture with a dynamic one in a bioreactor. To reproduce a physiological

372 displacement configuration, the stretching parameters of the bioreactor were chosen

373 consistently with the previous literature (Bosworth et al., 2014). At the end of the

374 cultures, the full-field XCT investigation permitted to successfully visualize the

375 fibroblasts grown on the external sheaths (Figure 3). Considering such complex and

376 three-dimensional nanofibrous scaffolds, acquiring XCT images was challenging. In

377 their work, Bradley et al. (Bradley et al., 2017) were able to visualize human fibroblasts

378 seeded on electrospun poly(lactide-co-glycolide) (PLGA) random microfibrous mats by

379 using a laboratory XCT scanner thanks to the micrometric cross-section of the fibers and

380 the different levels the X-rays attenuation between the PLGA and the cellular

381 component. In the case of the PLLA/Coll nanofibers instead, it is difficult to obtain

382 tomographic images fibers, due to the low absorption of the collagen of X-rays (Balint

383 et al., 2016; Zidek et al., 2016). This criticality is increased when the aim of the XCT

384 scan is to discriminate elements with a similar attenuation and dimensions, such as cells

385 and collagenous nanofibers. This aspect was fundamental for the XCT visualization of

386 fibroblasts. Due to their spread shape and the random arrangement of the nanofibers, 
387 fibroblasts detection on the electrospun sheath was clearly visible (Figure 3(B)).

388 Conversely, the identification of cells inside the internal bundles was not distinguishable

389 (Figure 3(AII) and 3(AIV)). This was mainly caused by the axially aligned nanofibers

390 and the thinner and elongated shape of the cells. Further optimization, especially in the

391 thresholding phase would possibly to allow the XCT detection of cells along the bundles

392 aligned nanofibers.

393 In order to overcome this limitation and to validate the XCT results, additional imaging

394 techniques such as fluorescence microscopy, SEM and histology were performed. The

395 combination of these imaging protocols confirmed that fibroblasts on the electrospun

396 sheath of the scaffolds adopt a different shape depending on the culture conditions

397 employed. Both on the static and dynamic specimens, the cells had a length that was one

398 order of magnitude longer than the other two dimensions. On the electrospun sheath of

399 the static specimens, cells were elongated along the circumference of the scaffolds with

400 a spread body, while in the dynamic ones, a prevalent axial orientation with thinner and

401 slender morphology was observed (Figures 3-5 and Figure 7).

402 Moreover, the SEM and histological investigations showed that fibroblasts were able to 403 penetrate inside the electrospun sheath, growing and aligning themselves in the direction 404 of the axially aligned nanofibers. In the dynamic specimens, the cells bodies were 405 slightly thinner and shorter (length $=21.4 \pm 8.5$ micrometers; thickness $=1.2 \pm 0.8$ 406 micrometers) compared to the static ones (length $=26.8 \pm 9.9$ micrometers; thickness $=$ $407 \quad 1.5 \pm 0.2$ micrometers) (Figures 4, 5 and Figure 7). These results were in accordance with 408 the previous studies on cell cultures carried out on PLLA/Coll electrospun bundles of 409 aligned nanofibers (Sensini \& Cristofolini, 2018; Sensini et al., 2018).

410 However, considering the different imaging investigations, the fibroblasts grown on the 411 sheath of the hierarchical scaffolds showed an unprecedented phenomenon compared to 
412 previous cell studies (Alshomer et al., 2018; Hampson et al., 2008; Sensini \&

413 Cristofolini, 2018). In fact, the circumferential alignment and elongation of cells grown

414 in the static condition was unexpected, even considering the slightly circumferential

415 alignment of the sheath nanofibers (Figure 2). Moreover, when cultured under dynamic

416 conditions, the sheath fibroblasts progressively elongated their shape trying to align

417 themselves to the axis of the hierarchical scaffolds. All these qualitative considerations

418 on cellular orientation were confirmed by the cellular Directionality analysis performed

419 on cells grown on the sheaths for the different conditions of culture (Figure 6). This

420 behavior can be probably ascribed to the combination of three factors: the

421 electrospinning production process of the sheath, the hydration and mechanical

422 component, and the crosslinking of the nanofibers. Firstly, the mechanism to produce

423 the sheath was proved to tune the level of compacting of the internal bundles of the

424 hierarchical scaffolds (WO 2018/229615 A1, 2018; Sensini et al., 2019; Sensini et al.,

425 2019). This effect causes a pre-tensioning of the sheath nanofibers and of the internal

426 bundles. Secondly, after immersion in the culture medium, the scaffolds absorbed the

427 liquid that likely resulted in swelling of the internal bundles inducing additional

428 stretching of the sheath. The combination of those two effects can explain the presence

429 of circumferential stresses, that could in turn drive the fibroblasts to change shape even

430 in static conditions. The progressive axial alignment of cells in the dynamic cultures

431 instead, could be explained by considering the effect of the collagen crosslinking. In

432 fact, it is possible that, during the crosslinking process, the nanofibers at the interface

433 between the sheath and the internal bundles could have been crosslinked together,

434 reducing their sliding. This could have caused a transmission of the axial load between

435 the bundles and sheath, producing an increment of the longitudinal stretch of the sheath

436 themselves, that induced the cells alignment. Both these effects, to the best of our 
437 knowledge, were completely unexplored so far and they need further investigations in

438 the near future increasing the sample size of the hierarchical scaffolds tested.

\section{Conclusion}

440 In this study a preliminary investigation on the change in fibroblasts morphology was

441 assessed by culturing them on electrospun hierarchical scaffolds in static and dynamic

442 conditions. The integration between XCT scans and gold-standard techniques such as

443 SEM, fluorescence microscopy and histology allowed the detection of the modifications

444 in the cell morphology and orientation. Considering the results, these electrospun

445 hierarchical scaffolds could be suitable for future in vivo animal study, permitting an

446 axial orientation of cells both on the electrospun sheath and the internal bundles when

447 stimulated with axial loads. Moreover, the improvement of the imaging protocols

448 developed in this study will be useful for the future development of correlative

449 microscopy workflows dedicated to similar electrospun materials.

\section{Acknowledgments}

451 The Italian Ministry of University and Research (MIUR) is acknowledged. The mobility

452 of Alberto Sensini was funded by the University of Bologna (Marco Polo grant). Type I

453 collagen was kindly provided by Kensey Nash Corporation d/b/a DSM Biomedical

454 (Exton, USA). The Zeiss Global Centre at the University of Portsmouth is greatly

455 acknowledged for the support in X-ray imaging and data post-processing. The project

456 was partially funded by the University of Portsmouth through a Research and Innovation

457 Development Fund. The authors greatly acknowledge CellScale and Jim Veldhuis for 458 technical training of Alberto Sensini in the use of the bioreactor. The authors

459 acknowledge Carlo Gotti and Marina Fichera for the help during the scaffolds 460 production and the imaging post processing. Marco Curto, Martino Pani and Robin 
461 Rumney were also gratefully acknowledged for the help and suggestions in the design

462 of the 3D-printed parts, design tables reviewing, and the cell culture planning. The

463 authors also gratefully acknowledge Sabrina Valente and Gianandrea Pasquinelli for the

464 useful suggestions and the use of the facilities during the histologic investigations.

\section{Figures}

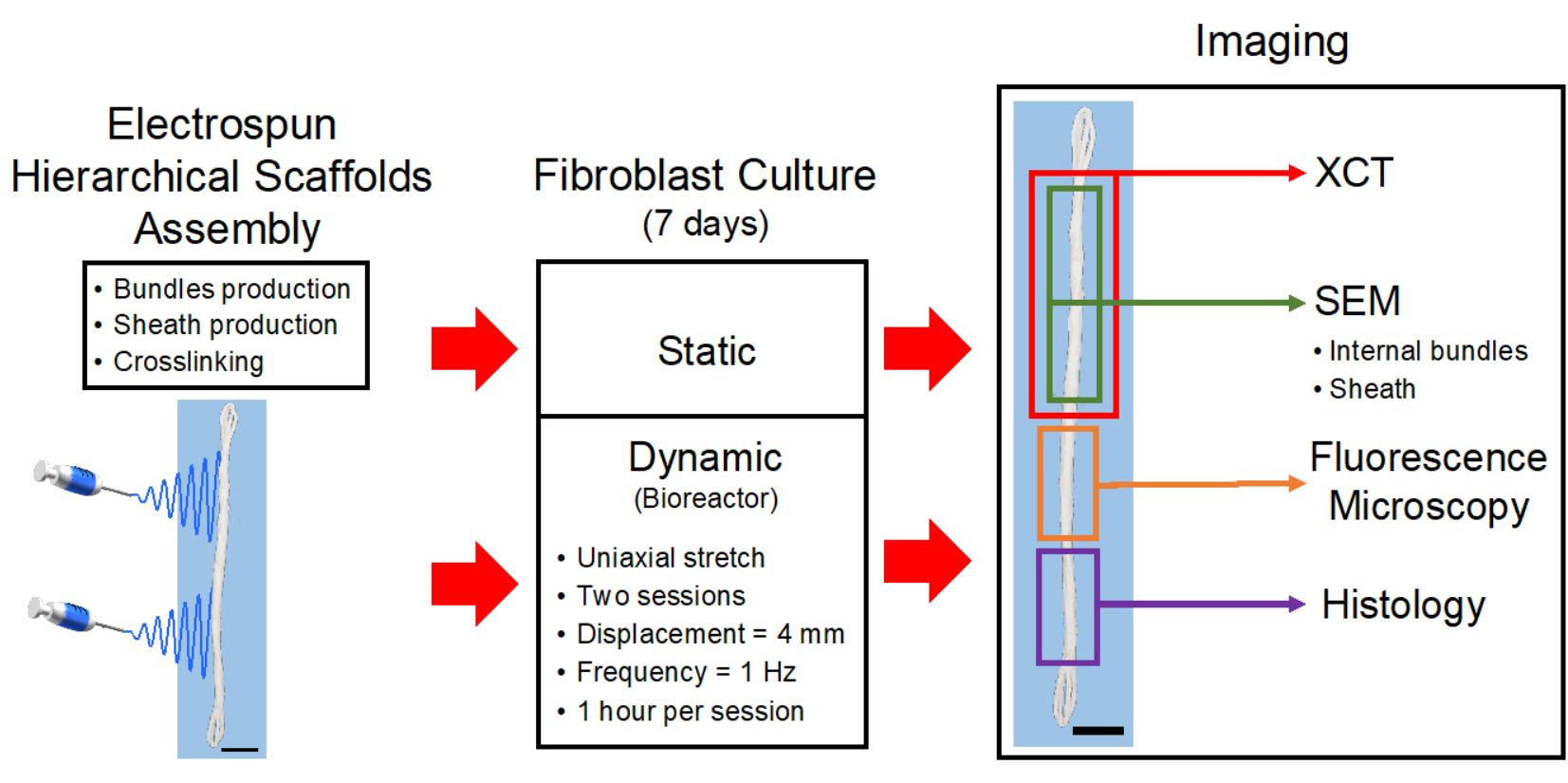

(A)

(B)

(C)

467 Fig. 1. Workflow of the experiment. (A) Electrospun hierarchical scaffolds assembly

468 (scale bar $=1 \mathrm{~mm}$ ). (B) Fibroblasts culture: two scaffolds were cultured in static

469 conditions, while other two with uniaxial sessions of stretching in a bioreactor. (C)

470 Scaffolds preparation for the different imaging investigations (scale bar $=1 \mathrm{~mm}$ ). 


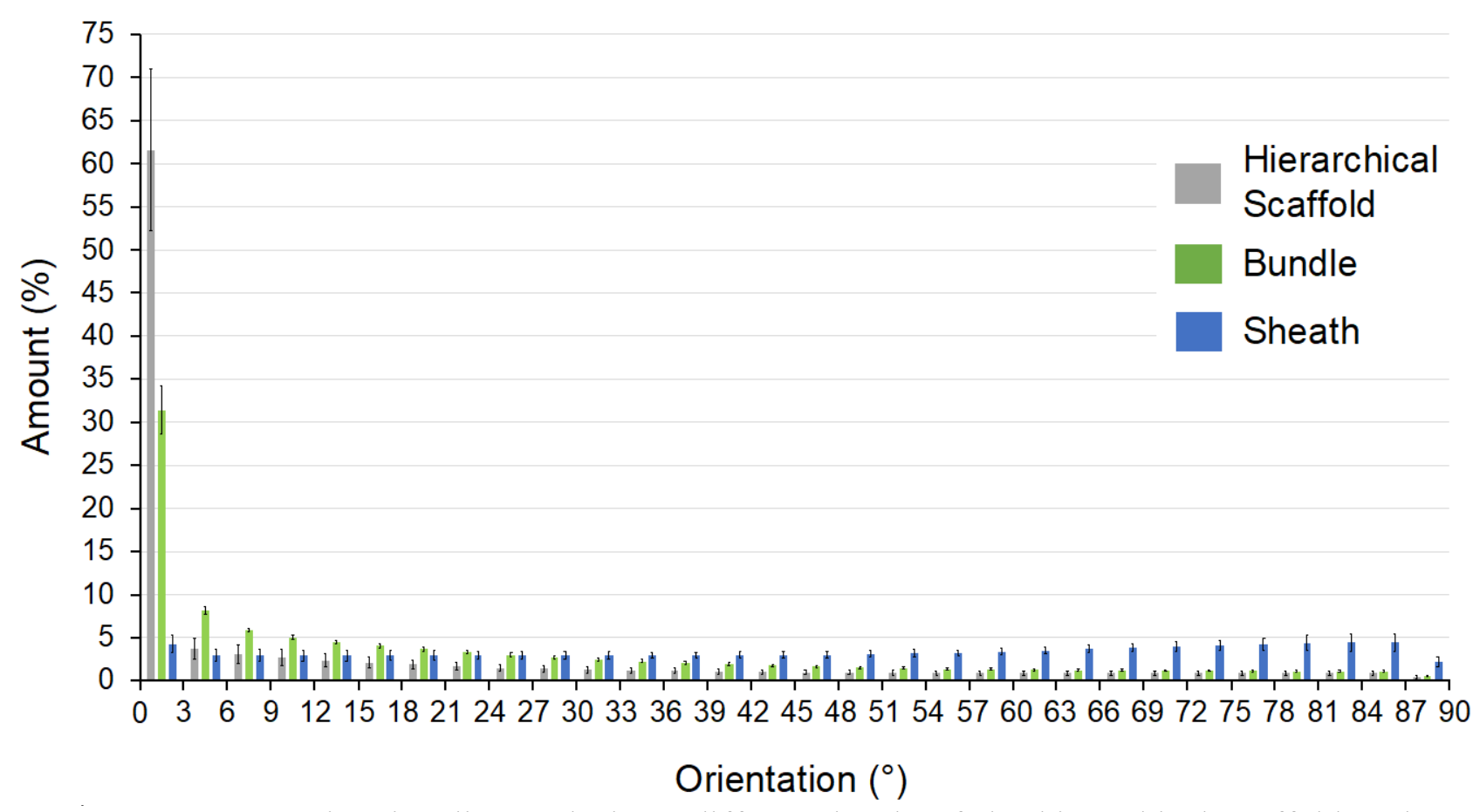

473 Fig. 2. Directionality analysis at different levels of the hierarchical scaffolds. The

474 directionality histograms show the comparison between: the alignment of the bundles

475 inside the hierarchical scaffold (gray bars), the distribution of nanofibers in the different

476 directions for the bundle (green bars) and on the electrospun sheath (blue bars). An angle

477 of $0^{\circ}$ means that the nanofibers were aligned with the longitudinal axis of the

478 hierarchical scaffold, an angle of $90^{\circ}$ means that the nanofibers were perpendicular to it.

479 Mean and standard deviation between images of the same specimen are plotted. 


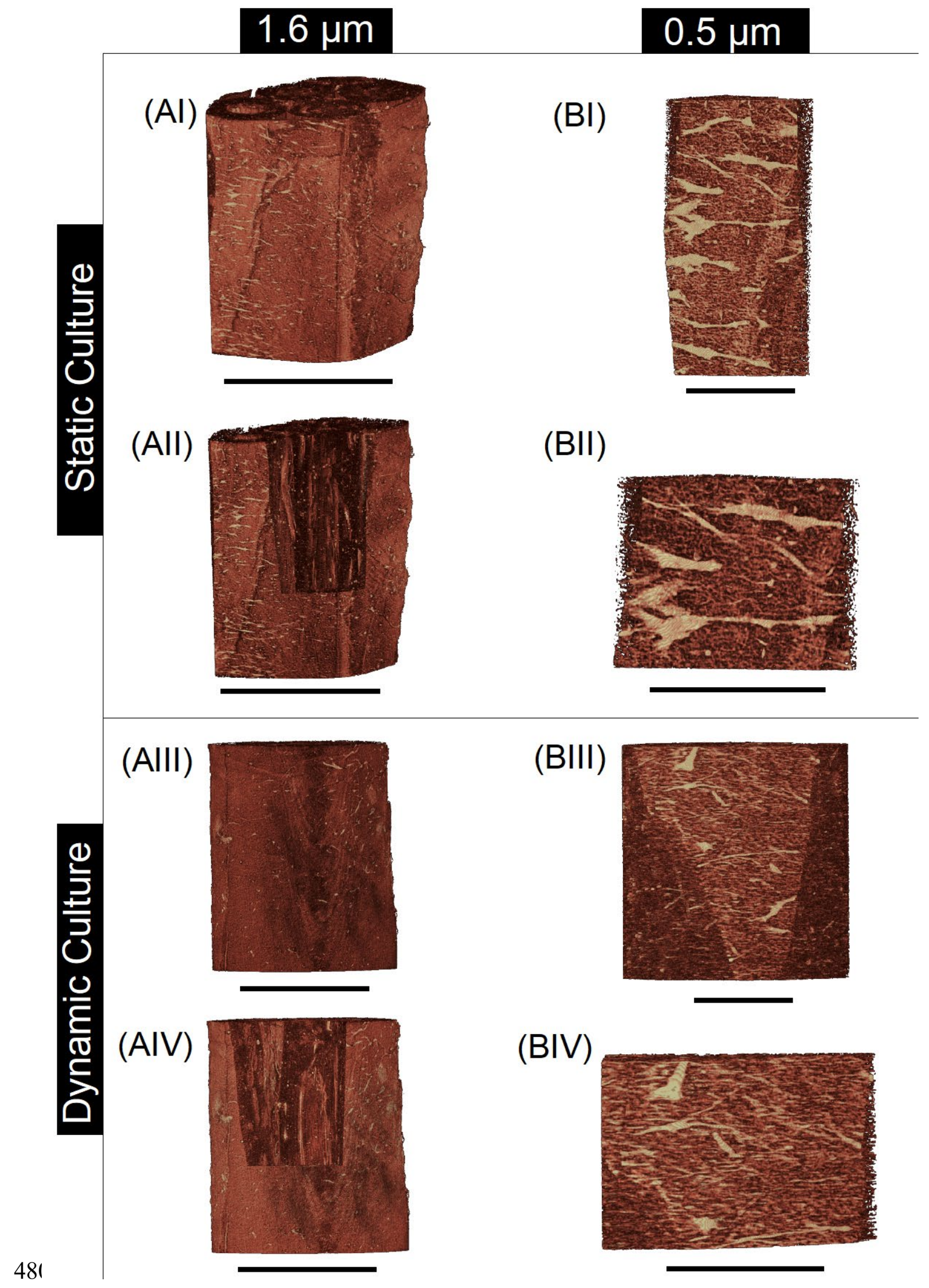

481 Fig. 3. XCT images of fibroblasts cultured onto the hierarchical scaffolds in static (I,

482 II) and dynamic (III, IV) conditions. (AI, III) Overview of the scaffolds; (AII, IV) 
483 superficial crop showing the internal bundles (voxel size $=1.6$ micrometers; scale bar

$484=1 \mathrm{~mm}$ ). (BI, III) Overview of fibroblasts on the external sheath; (AII, IV) zoom on the

485 fibroblasts (voxel size $=0.5$ micrometers; scale bar $=200$ micrometers).

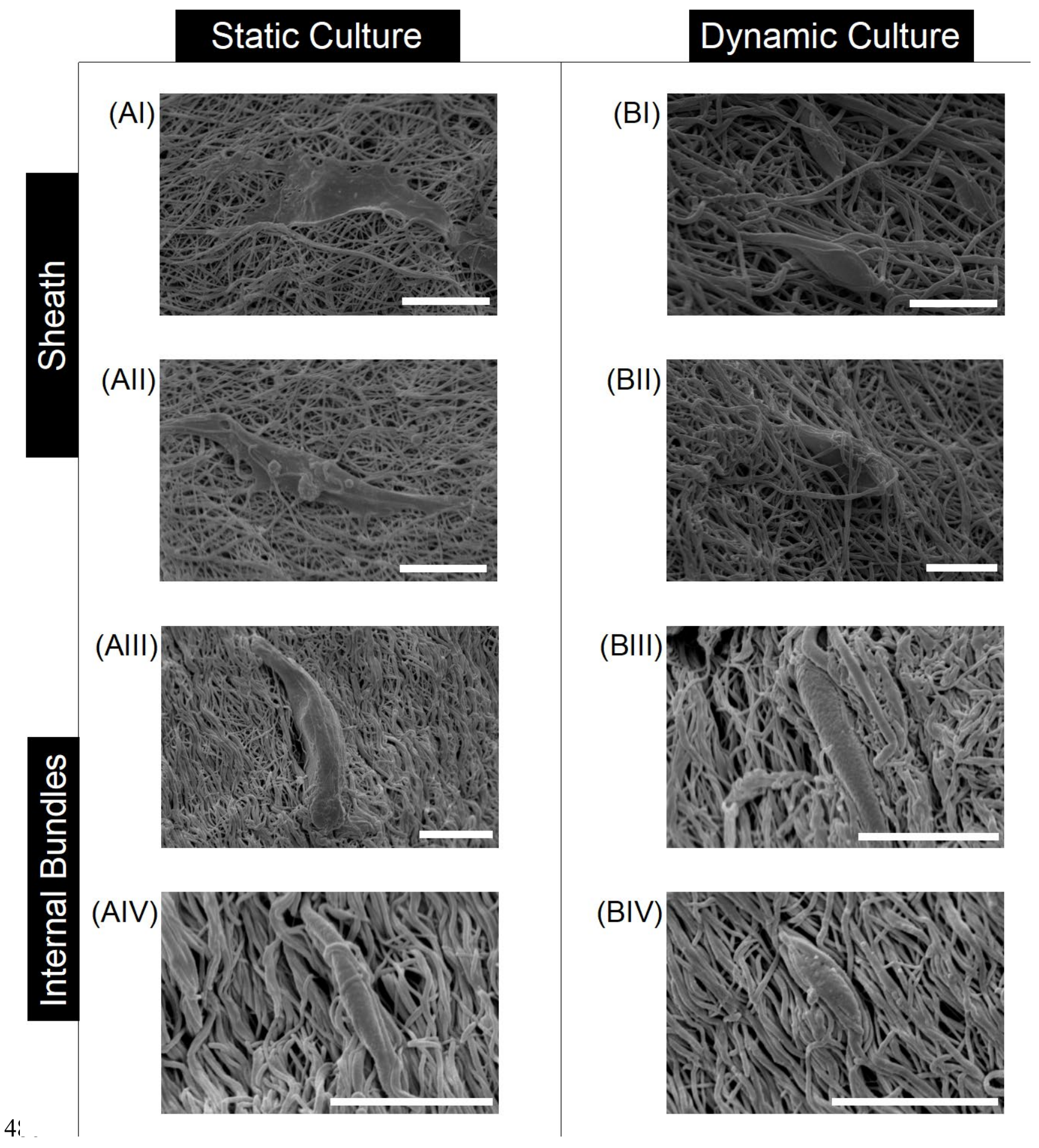


Fig. 4. SEM images of fibroblasts cultured onto the hierarchical scaffolds in static (A)

488 and dynamic (B) conditions (scale bar $=10$ micrometers). (I-II) SEM images of the

489 fibroblasts on the electrospun sheath; (III-IV) SEM fibroblasts on the internal bundles.

\section{Static Culture}

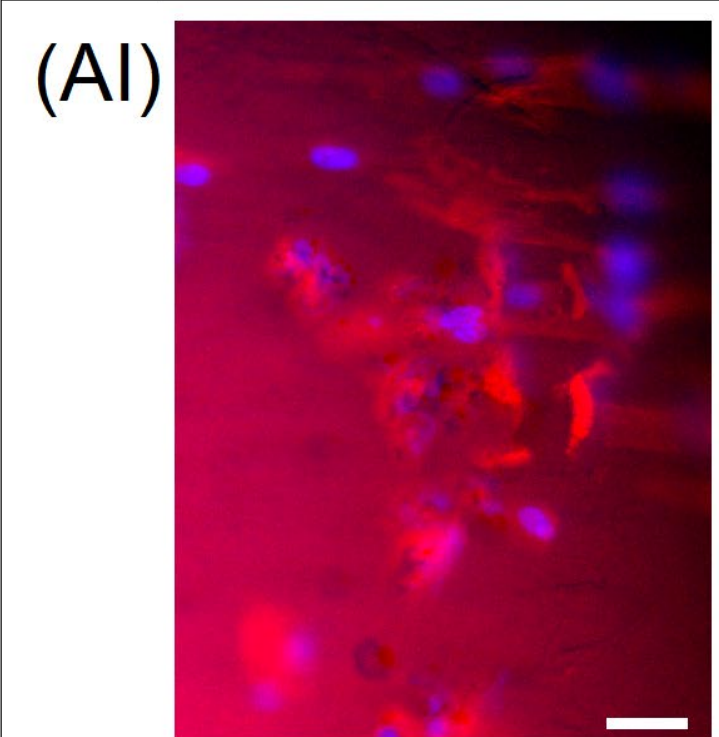

(AII)
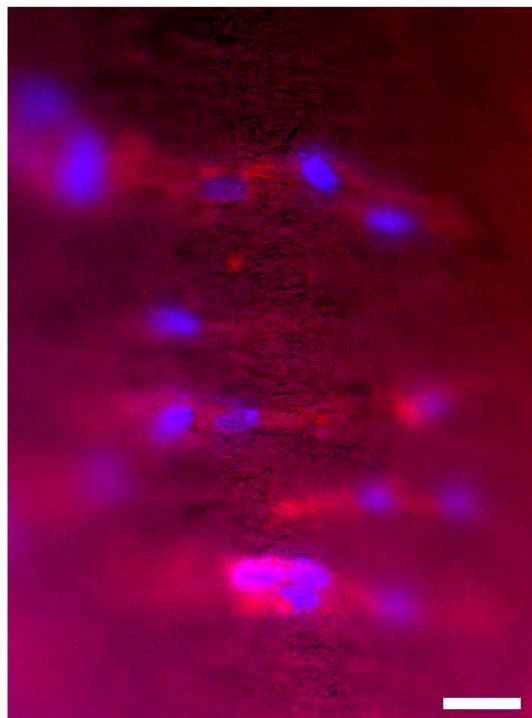

4

491 Fig. 5. Fluorescence images of fibroblasts onto the hierarchical scaffolds sheath in static

492 (A) and dynamic (B) conditions (scale bar $=30$ micrometers). 


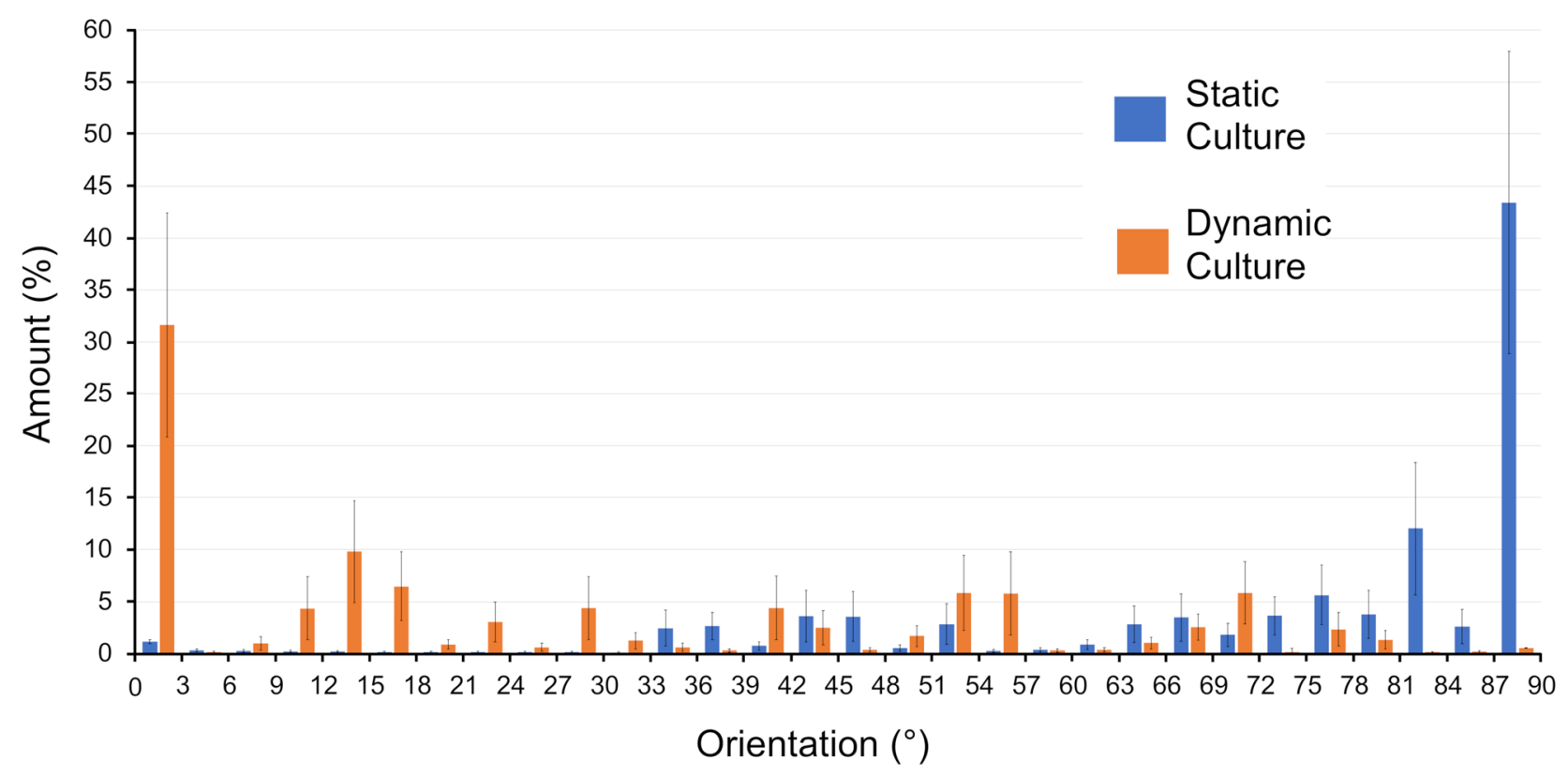

494 Fig. 6. Directionality analysis of cells grown on the electrospun sheaths (based on the 495 orientation of the cells nuclei) in static and dynamic conditions of culture. An angle of $4960^{\circ}$ means that the cells were aligned with the longitudinal axis of the hierarchical 497 scaffold, an angle of $90^{\circ}$ means that the cells were perpendicular to it. Mean and standard 498 deviation between images of the static and dynamic specimens are plotted. 


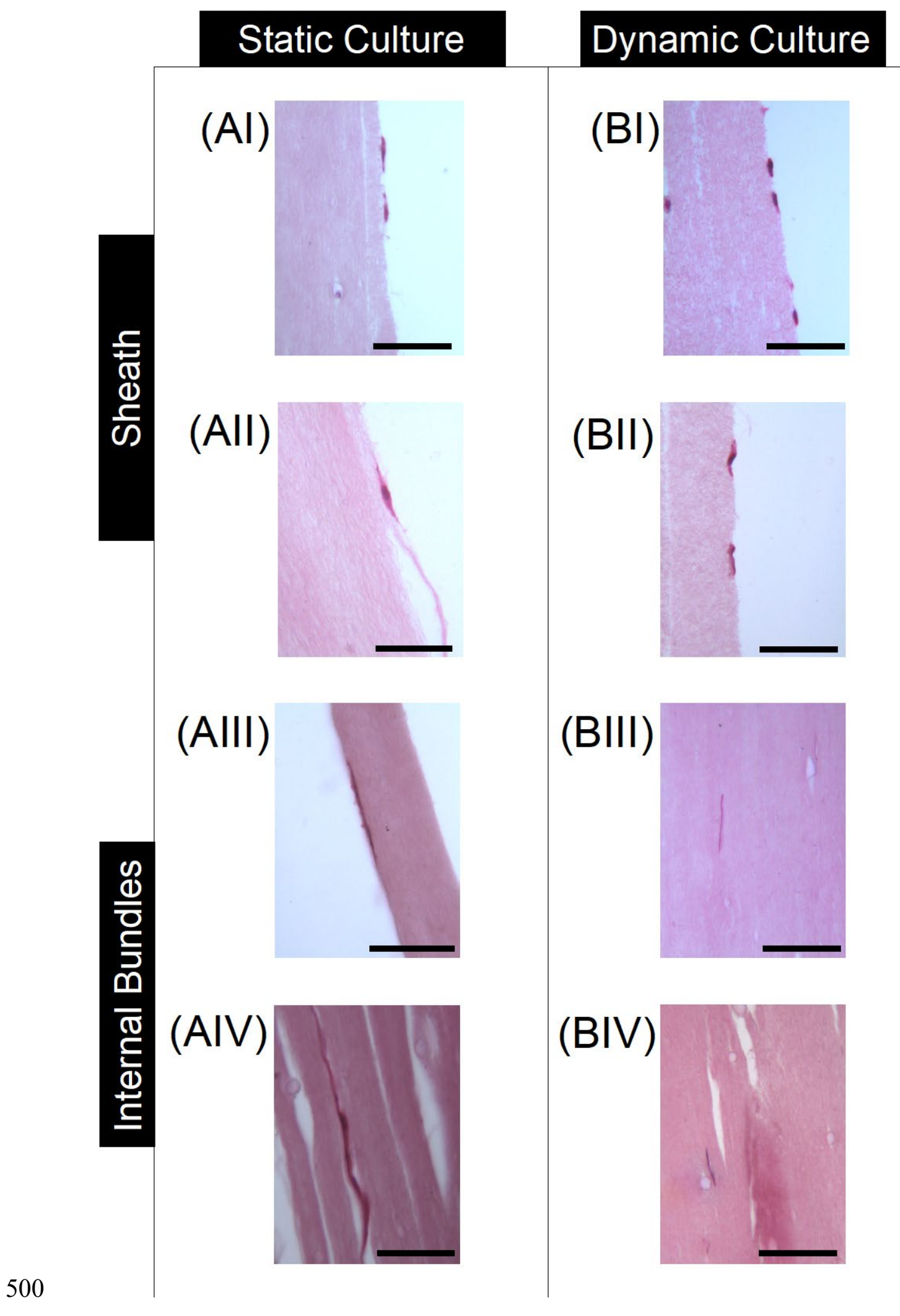

501 Fig. 7. Histological investigation on the hierarchical scaffolds cultured in static (A) and 502 dynamic (B) conditions (scale bar $=50$ micrometers). (I-II) Zoom-in on the fibroblasts 
503 on the electrospun sheath; (III-IV) images of the elongated fibroblasts on the aligned 504 nanofibers of the internal bundles.

505 


\section{References:}

Alshomer, F., Chaves, C., \& Kalaskar, D. M. (2018). Advances in Tendon and Ligament Tissue Engineering: Materials Perspective. J. Mater., 2018, 1-18. https://doi.org/10.1155/2018/9868151

Balint, R., Lowe, T., \& Shearer, T. (2016). Optimal contrast agent staining of ligaments and tendons for X-ray computed tomography. PLoS One, 11(4). https://doi.org/10.1371/journal.pone.0153552

Bosworth, L. A., Rathbone, S. R., Bradley, R. S., \& Cartmell, S. H. (2014). Dynamic loading of electrospun yarns guides mesenchymal stem cells towards a tendon lineage. J. Mech. Behav. Biomed. Mater., 39, 175-183. https://doi.org/10.1016/j.jmbbm.2014.07.009

Bosworth, L. A., \& Downes, S. (2011). Electrospinning for Tissue Regeneration. (Lucy A. Bosworth \& S. Downes, Eds.). Cambridge: Woodhead Publishing. https://doi.org/10.1016/B978-1-84569-741-9.50001-X

Bradley, R. S., Robinson, I. K., \& Yusuf, M. (2017). 3D X-Ray Nanotomography of Cells Grown on Electrospun Scaffolds. Macromol. Biosci., 17(2), 1-8. https://doi.org/10.1002/mabi.201600236

Cheng, M. T., Shih, Y. R. V., \& Lee, O. K. (2015). Tendon and Ligament Tissue Engineering. In A. Vishwakarma, P. Sharpe, S. Shi, \& M. Ramalingam (Eds.), Stem Cell Biology and Tissue Engineering in Dental Sciences (42, 553-565), Elsevier Inc. https://doi.org/https://doi.org/10.1016/B978-0-12-397157-9.00076$\mathrm{X}$

Denchai, A., Tartarini, D., \& Mele, E. (2018). Cellular Response to Surface Morphology: Electrospinning and Computational Modeling. Front. Bioeng. Biotechnol., 6(October), 1-11. https://doi.org/10.3389/fbioe.2018.00155 Goulet, F., Auger, F. A., Cloutier, R., Lamontagne, J., Simon, F., Chabaud, S., Germain, L., \& Hart, D. A. (2014). Tendons and Ligament Tissue Engineering. In R. Lanza, R. Langer, \& J. Vacanti (Eds.), Principles of Tissue Engineering (59, 1275-1287). Elsevier Inc. https://doi.org/10.1016/B978-0-12-398358-9.00059-8

Hampson, K., Forsyth, N. R., El Haj, A., \& Maffulli, N. (2008). Tendon Tissue Engineering. In N. Ashammakhi, R. Reis, \& F. Chiellini (Eds.), Topics in Tissue Engineering (3, 1-21). https://www.oulu.fi/spareparts/ebook_topics_in_t_e_vol4/abstracts/hampson.pdf Kannus, P. (2000). Structure of the tendon connective tissue. Scand. J. Med. Sci. Sport., 10(6), 312-320. https://doi.org/10.1034/j.1600-0838.2000.010006312.x Kastelic, J., Galeski, A., \& Baer, E. (1978). The Multicomposite Structure of Tendon. Connect. Tissue Res., 6(1), 11-23. https://doi.org/10.3109/03008207809152283

Kuo, C. K., Marturano, J. E., \& Tuan, R. S. (2010). Novel strategies in tendon and ligament tissue engineering: Advanced biomaterials and regeneration motifs. Sport. Med. Arthrosc. Rehabil. Ther. Technol., 2(20), 1-14.

Leferink, A. M., Van Blitterswijk, C. A., \& Moroni, L. (2016). Methods of Monitoring Cell Fate and Tissue Growth in Three-Dimensional Scaffold-Based Strategies for In Vitro Tissue Engineering. Tissue Eng. Part B Rev., 22(4), 1-19. https://doi.org/10.1089/ten.teb.2015.0340

Liu, Z. (1991). Scale space approach to directional analysis of images. Appl. Opt., 30(11), 1369-1373. https://doi.org/https://doi.org/10.1364/AO.30.001369

Murphy, W., Black, J., \& Hastings, G. (2016). Handbook of Biomaterial Properties. (W. Murphy, J. Black, \& G. Hastings, Eds.) (Second). Springer. https://doi.org/10.1007/978-1-4939-3305-1

Schindelin, J., Arganda-Carreras, I., Frise, E., Kaynig, V., Longair, M., Pietzsch, T., 
Preibish, S., Rueden, C., Saalfeld, S., Schmid, B., Tinevez, J. Y., White, D. J., Hartenstein, V., Eliceiri, K., Tomancak, P., \& Cardona, A. (2012). Fiji: an opensource platform for biological-image analysis. Nat. Methods, 9(7), 676-682. https://doi.org/10.1038/nmeth.2019

Schneider, C. A., Rasband, W. S., \& Eliceiri, K. W. (2012). NIH Image to ImageJ: 25 years of image analysis. Nat. Methods, 9(7), 671-675. https://doi.org/10.1038/nmeth.2089

Sensini, A., Cristofolini, L., Focarete, M. L., Belcari, J., Zucchelli, A., Kao, A., \& Tozzi, G. (2018). High-resolution x-ray tomographic morphological characterisation of electrospun nanofibrous bundles for tendon and ligament regeneration and replacement. J. Microsc., 272(3), 196-206. https://doi.org/10.1111/jmi.12720

Sensini, A., \& Cristofolini, L. (2018). Biofabrication of Electrospun Scaffolds for the Regeneration of Tendons and Ligaments. Materials (Basel)., 11(10), 1963. https://doi.org/10.3390/ma11101963

Sensini, A., Cristofolini, L., Gualandi, C., Focarete, M. L., Belcari, J., \& Zucchelli, A. (2018). WO 2018/229615 A1.

Sensini, A., Gotti, C., Belcari, J., Zucchelli, A., Focarete, M. L., Gualandi, C., Todaro, I., Kao, A. P., Tozzi, G., \& Cristofolini, L. (2019). Morphologically bioinspired hierarchical Nylon 6.6 electrospun assembly recreating the structure and performance of tendons and ligaments. Med. Eng. Phys. https://doi.org/10.1016/j.medengphy.2019.06.019

Sensini, A., Gualandi, C., Cristofolini, L., Tozzi, G., Dicarlo, M., Teti, G., MattioliBelmonte, M., \& Focarete, M. L. (2017). Biofabrication of bundles of poly(lactic acid)-collagen blends mimicking the fascicles of the human Achille tendon. Biofabrication, 9(1). https://doi.org/10.1088/1758-5090/aa6204

Sensini, A., Gualandi, C., Focarete, M. L., Belcari, J., Zucchelli, A., Boyle, L., Reilly, G. C., Kao, A. P., Tozzi, G., \& Cristofolini, L. (2019). Multiscale hierarchical bioresorbable scaffolds for the regeneration of tendons and ligaments. Biofabrication, 11(3), 35026. https://doi.org/10.1088/1758-5090/ab20ad

Sensini, A., Gualandi, C., Zucchelli, A., Boyle, L., Kao, A. P., Reilly, G. C., Tozzi, G., Cristofolini, L., \& Focarete, M. L. (2018). Tendon Fascicle-Inspired Nanofibrous Scaffold of Polylactic acid/Collagen with Enhanced 3D-Structure and Biomechanical Properties. Sci. Rep., 8(1), 1-15. https://doi.org/https://doi.org/10.1038/s41598-018-35536-8

Tseng, L. F., Mather, P. T., \& Henderson, J. H. (2013). Shape-memory-actuated change in scaffold fiber alignment directs stem cell morphology. Acta Biomater., 9(11), 8790-8801. https://doi.org/10.1016/j.actbio.2013.06.043

Wu, S., Wang, Y., Streubel, P. N., \& Duan, B. (2017). Living nanofiber yarn-based woven biotextiles for tendon tissue engineering using cell tri-culture and mechanical stimulation. Acta Biomater., 62, 102-115. https://doi.org/10.1016/j.actbio.2017.08.043

Xu, Y., Dong, S., Zhou, Q., Mo, X., Song, L., Hou, T., Wu J., Li, S., Li, Y., Li, P., Gan, Y., \& Xu, J. (2014). The effect of mechanical stimulation on the maturation of TDSCs-poly(L-lactide-co-e-caprolactone)/collagen scaffold constructs for tendon tissue engineering. Biomaterials, 35(9), 2760-2772. https://doi.org/10.1016/j.biomaterials.2013.12.042

Youngstrom, D. W., \& Barrett, J. G. (2016). Engineering tendon: Scaffolds, bioreactors, and models of regeneration. Stem Cells Int., 2016, 1-12. https://doi.org/10.1155/2016/3919030 
606

607

608

609

610
Zidek, J., Vojtova, L., Abdel-Mohsen, A. M., Chmelik, J., Zikmund, T., Brtnikova, J., Jakubicek, R., Zubal, L., Jan, J., \& Kaiser, J. (2016). Accurate micro-computed tomography imaging of pore spaces in collagen-based scaffold. J. Mater. Sci. Mater. Med., 27(6), 1-18. https://doi.org/10.1007/s10856-016-5717-2 